\title{
Downregulated SOCS1 expression activates the JAK1/STAT1 pathway and promotes polarization of macrophages into M1 type
}

\author{
YAN-BING LIANG ${ }^{*}$, HAO TANG ${ }^{*}$, ZHI-BIN CHEN, LI-JIN ZENG, JING-GUO WU, \\ WEN YANG, ZHEN-YU LI and ZHONG-FU MA
}

Department of General Internal Medicine, The First Affiliated Hospital, Sun Yat-sen University, Guangzhou, Guangdong 510080, P.R. China

Received June 25, 2016; Accepted April 25, 2017

DOI: $10.3892 / \mathrm{mmr} .2017 .7384$

\begin{abstract}
Macrophage polarization is flexible, and involves in different signaling pathways and various transcription factors. Suppressor of cytokine signaling (SOCS) is an important inhibitor of cytokine signaling pathways and also a key physiological regulator for natural and acquired immunity systems. Following transfection of SOCS1 shorthairpin(sh)RNA into mouse macrophage cells, reverse transcription-quantitative polymerase chain reaction demonstrated that the mRNA levels of Janus kinase (JAK)1 and signal transducer and activator of transcription (STAT)1 increased significantly. In addition, western blotting indicated that JAK1, STAT1 and p-STAT1 expression was significantly enhanced. Fludarabine can inhibit phosphorylation of STAT1 and SOCS1 expression. When fludarabine was added and SOCS1 shRNA was transfected, the inhibition of fludarabine was weakened, and p-STAT1 expression was upregulated. Flow cytometry detection indicated that, following the downregulation of SOCS1 expression, M1-type cells significantly increased, but the proportion of M2-type cells did not change significantly. Fludarabine can reduce the effect of SOCS1 shRNA on promoting M1-type cell polarization, and macrophages can polarize into both M1 and M2 phenotypes. Further ELISA results presented that, when downregulating SOCS1 expression, interleukin (IL)-4 and IL-10 expression was both downregulated, and tumor necrosis factor (TNF)- $\alpha$ and interferon (IFN)- $\gamma$ expression was significantly upregulated. When adding fludarabine or injecting with the traditional Chinese medicine Xuebijing, IL-4 and IL-10 expression was both significantly upregulated, and
\end{abstract}

Correspondence to: Dr Zhong-Fu Ma, Department of General Internal Medicine, The First Affiliated Hospital, Sun Yat-sen University, 58 Zhongshan Second Road, Guangzhou, Guangdong 510080, P.R. China

E-mail: 1362670488@qq.com

*Contributed equally

Key words: suppressor of cytokine signaling-1, Janus kinase 1, signal transducer and activator of transcription 1, macrophage
TNF- $\alpha$ and IFN- $\gamma$ expression was significantly downregulated. When adding fludarabine and downregulating SOCS1, IL-4, IL-10, TNF- $\alpha$ and IFN- $\gamma$ expression presented no significant changes. The above results indicated that, when SOCS1 expression is downregulated, it will activate the JAK1/STAT1 pathway, and thereby promote the polarization of macrophages into M1 type. The findings are of great importance for understanding occurrence, development and treatment of various immune-related diseases.

\section{Introduction}

Macrophages are important phagocytic and antigen-presenting cells in the body and they serve an important role in handling external pathogenic microorganisms and endogenous danger signals. Macrophages are widely distributed in various tissues and organs and are important in maintaining homeostasis, body defense, regulating inflammation and promoting wound healing (1).

As important immune cells in human body, macrophages serve an important role in antigen presentation and inflammatory response. A recent study demonstrated that macrophages are a group of heterogeneous and flexible cells with diverse and varied immune functions, and they can polarize into different phenotypes in different microenvironments or under different stimuli, playing different roles in inflammatory response of tissues (2). Depending on the activation and immune functions, they can be divided into the classically activated type (M1 type) and the alternatively activated type (M2 type). M1 type macrophages are activated by $\mathrm{T}$ helper (h) 1 cytokines of helper $\mathrm{T}$ lymphocytes, such as interferon (IFN), tumor necrosis factor (TNF) and bacterial lipopolysaccharides (LPS), and the activated cells can induce the expression of pro-inflammatory cytokines, such as interleukin (IL)-6, TNF- $\alpha$ and IL- $1 \beta$, resist pathogen invasion, and participate in the inflammatory response, but they can also cause body injury, which is manifested as high antigen-presenting ability (3). M2 type macrophages are activated by Th2 cytokines and, following activation, they can induce high expression of anti-inflammatory cytokines including IL-10, transforming growth factor $\beta$ and arginase, and low expression of pro-inflammatory cytokines, thereby inhibiting the inflammatory response, protecting surrounding tissues from the harm caused by immune response 
and promoting repair of tissue injuries (4). Therefore, a deep study into the internal mechanism of macrophages polarity differentiation will be of great importance for a better understanding occurrence, development and treatment of various immune-related diseases.

The suppressor of cytokine signaling (SOCS) family is a collection of negative regulatory factors that are generated by cells and can block cytokine signaling in feedback (5). As one important member of the family, SOCS1 is involved in various cytokine signal transductions and the differentiation of immune cells, and serves an important role in innate and adaptive immune response. As an important determinant for the activity and function of differentiated macrophages, SOCS1 is not only a feedback inhibitor of inflammation, but is also an important molecular switch that can effectively regulate different aspects of macrophage balance by regulating crucial signaling pathways (6). In the case of tissue inflammation, macrophages will have high expression of SOCS1 or SOCS3, but the probability of simultaneous expression of both is small. By using IFN- $\gamma$ or LPS to culture mouse bone marrow-derived macrophages, they could express SOCS1 and SOCS3, yet following stimulation with IL-4, macrophages only expressed SOCS1 $(6,7)$. In contrast, when both IFN- $\gamma$ and LPS were used for culturing, expression of SOCS1 in macrophages was inhibited, and these cells gradually polarized into M1 macrophages that only express SOCS3. Following the knock out of SOCS3 in macrophages, culturing with IFN- $\gamma$ and LPS led to an upregulation of SOCS1 expression. The cells restored the reactivity for IL-4, thereby polarizing into M2 macrophages and inhibiting the generation of M1 macrophages and inflammatory mediators. The results demonstrated that SOCS3 is of great importance for the activation of M1 macrophages, while SOCS1 can control macrophages' reactivity for IFN- $\gamma$, as well as signaling pathways of TLR4 and TLR9 activation; it is also an endogenous inhibitor of the STAT1 pathway. Upregulated SOCS1 expression can promote the polarization of macrophages into M2 type, indicating that SOCS1 may be involved in the polarization process into M2 type macrophages (8-10).

Although there currently is a certain understanding of SOCS1, its specific mechanism remains to be further explored so as to better guide our clinical practice. In addition, Xuebijing (XBJ) injection is an intravenous preparation made from traditional Chinese medicines. Previous studies and clinical trials have indicated that it is a good treatment of SIRS/MODS (systemic inflammatory response syndrome/multiple organ dysfunction syndrome) $(11,12)$. In addition, current fundamental research has suggested that it could restrain the release of inflammatory mediators, eliminate endotoxin and reduce the mortality of septic animals and patients $(11,12)$. Nevertheless, its effect on STATs and SOCSs has not been explored until now. Thus, the present study will make a discussion to provide a clinical basis for determining the mechanism of Xuebijing.

\section{Materials and methods}

Isolation and culture of mouse macrophages. Animal care and use followed the ethical guidelines of the Chinese Council on Animal Care and were reviewed and approved by the Institutional Animal Care and Use Committee. The mice (purchased from the Institute of Laboratory Animal Sciences,
Chinese Academy of Medical Sciences and Peking Union Medical College, Beijing, China) were sacrificed by cervical dislocation. A total of $5 \mathrm{ml}$ chilled RPMI 1640 medium (Gibco; Thermo Fisher Scientific, Inc., Waltham, MA, USA) was intraperitoneally injected. At $5 \mathrm{~min}$, a pipette was used to draw peritoneal lavage fluid, and the lavage was repeated once. The lavage fluid recycled twice was centrifuged at $4^{\circ} \mathrm{C}$, $8,000 \mathrm{x}$ g, and the obtained cell pellet was washed twice with pre-cooled RPMI1640 medium, and pre-cooled RPMI1640 medium (penicillin $100 \mathrm{U} / \mathrm{ml}$, streptomycin $100 \mu \mathrm{g} / \mathrm{ml}$ and $10 \%$ FBS) was added for resuspension. The cells were inoculated in $25 \mathrm{~mm}^{2}$ culture flasks (BD Biosciences, Franklin Lakes, $\mathrm{NJ}$, USA) and incubated at $37^{\circ} \mathrm{C}$ with $5 \% \mathrm{CO}_{2}$. Following $4 \mathrm{~h}$ incubation, the medium was changed and was rinsed with RPMI1640 medium twice, non-adherent cells were discarded and the adherent cells obtained were monolayer macrophages.

Construction and transfection of short hairpin (sh)RNA vector. shRNA targeting SOCS1 was synthesized, and was cloned into pSilencer 2.1-U6 neo vector (Qiagen GmbH, Hilden, Germany) following double enzyme digestion by BamHI and HindIII (Takara Biotechnology Co., Ltd., Dalian, China). The cells were seed into a six-well plate at $1 \times 10^{5}$ cells $/ \mathrm{ml}$ and incubated for $24 \mathrm{~h}$. The plasmid transfection was carried out following the instruction of Lipofectamine 2000 (Invitrogen; Thermo Fisher Scientific, Inc.) with a concentration of $4 \mu \mathrm{g} /$ well. Following transfection, fludarabine (2 $\mu \mathrm{M}$; Selleck Chemicals, Shanghai, China) or Xuebijing injection $(50 \mathrm{mg} / \mathrm{ml}$; Tianjin Chase Sun Pharmaceutical Co., Ltd., Tianjin, China) was added, and cells were cultured for $48 \mathrm{~h}$ prior to being analyzed by reverse transcription-quantitative polymerase chain reaction (RT-qPCR) and western blotting.

$R T-q P C R$. Total RNA was extracted with TRIzol (Invitrogen; Thermo Fisher Scientific, Inc.) according to the manufacturer's instruction, and then reverse transcription was performed with One Step SYBR PrimeScript RT-PCR kit (Takara Biotechnology Co., Ltd.). The reaction was performed with ABI PRISM 7500 Real-Time PCR system (Applied Biosystems; Thermo Fisher Scientific, Inc.) at $42^{\circ} \mathrm{C}$ for $5 \mathrm{~min}, 95^{\circ} \mathrm{C}$ for $10 \mathrm{sec}$; then at 40 cycles of $95^{\circ} \mathrm{C}$ for $5 \mathrm{sec}, 55^{\circ} \mathrm{C}$ for $30 \mathrm{sec}$ and $72^{\circ} \mathrm{C}$ for $30 \mathrm{sec}$. Primers used for RT-qPCR are presented in Table I. Three independent experiments were conducted for each sample. Data were analyzed by comparing the $2^{-\Delta \Delta \mathrm{Cq}}$ value (13).

Western blotting. Total cellular proteins were extracted by incubating cells in radioimmunoprecipitation assay buffer with protease inhibitors (Pierce; Thermo Fisher Scientific, Inc.). The protein concentrations in the lysates were determined by Quick Start Bradford Protein assay (Bio-Rad Laboratories, Inc., Hercules, CA, USA). SDS-PAGE was conducted using $12 \%$ gels (Bio-Rad Laboratories, Inc.) loading equal amount of proteins $(20 \mu \mathrm{g})$ per lane. Following electrophoresis, separated proteins were transferred to a polyvinylidene difluoride membrane (Pierce; Thermo Fisher Scientific, Inc.) and blocked with 5\% non-fat milk. Following this, the membranes were incubated with anti-SOCS1 antibody (dilution, 1:500; cat. no. ab62584), anti-JAK1 antibody (dilution, 1:400; cat. no. ab133666), anti-STAT1 antibody (dilution, 1:500; cat. no. ab99415), anti-p-STAT1 antibody (dilution, 1:500; cat. no. ab109461) and 
Table I. Primers for reverse transcription-quantitative polymerase chain reaction.

\begin{tabular}{ll}
\hline Primer & \multicolumn{1}{c}{ Sequence (5'-3') } \\
\hline JAK1-m-F & TCAAACCTGTGTCTCGCTCT \\
JAK1-m-R & ACGCTGTTAGTTTTCTGTGTCAG \\
SOCS1-m-F & CTCCTTGGGGTCTGTTGGC \\
SOCS1-m-R & GCGTGCTACCATCCTACTCG \\
STAT1-m-F & GACCTGTCATCCCGCAGAGA \\
STAT1-m-R & GGAGCAGAGCTGAAACGACC
\end{tabular}

F, forward; R, reverse.

anti-GAPDH antibody (dilution, 1:1,000; cat. no. ab181603; all from Abcam, Cambridge, MA, USA) in 5\% non-fat milk overnight at $4^{\circ} \mathrm{C}$, and then goat anti-rabbit IgG monoclonal antibody (dilution, 1:5,000; cat. no. sc-2004; Santa Cruz Biotechnology, Inc., Dallas, TX, USA) conjugated with horseradish peroxidase was incubated with the membranes for $1 \mathrm{~h}$ at room temperature. Protein bands were detected using the West Femto system (Pierce; Thermo Fisher Scientific, Inc.), and gray values of the bands were measured by Gel-Pro Analyzer software version 6.3 (Media Cybernetics, Rockville, MD, USA).

Flow cytometry. Following digesting macrophages with trypsin, RPMI1640 medium was added and cells were collected by pipetting, then the cells were centrifuged at 6,000 $\mathrm{x} g$ for 5 min at room temperature and washed once with PBS. PBS was used for resuspension, and fluorescein isothiocyanate-labeled CD206 antibody (dilution, 1:10; cat. no. EL921283; EterLife, London, UK) was added, and the cells were incubated in the dark for $30 \mathrm{~min}$. Following this, the phycoerythrin-labeled anti-mouse CD197 (CCR7) (1 $\mu$ g; cat. no. 12-1971-63; eBioscience, Inc., San Diego, CA, USA) was added, and cells were incubated for $30 \mathrm{~min}$. Following washing twice with PBS, and resuspended by $\mathrm{PBS}$, the cells were tested on BD Accuri ${ }^{\mathrm{TM}} \mathrm{C6}$ flow cytometer (BD Biosciences). The negative control (NC) group was transfected with an empty pSilencer 2.1-U6 neo vector. The control group was not transfected.

ELISA. IL-4 (cat. no. M4000B), IL-10 (cat. no. M1000B), TNF- $\alpha$ (cat. no. MTA00B) and IFN- $\gamma$ (cat. no. MIF00) were detected according to the manufacturer's protocol of the ELISA kits (R\&D Systems China Co., Ltd. (Shanghai, China).

Statistical analysis. SPSS statistical software (version, 17.0; SPSS, Inc., Chicago, IL, USA) was used for data processing, and measurement data were presented as mean \pm standard deviation and processed with t-test. Differences were analyzed by non-parametric statistical analysis (Mann-Whitney U tests) between control and treated groups, $\mathrm{P}<0.05$ was considered to indicate a statistically significant difference.

\section{Results}

SOCS1 shRNA screening. Studies have suggested that SOCS1 upregulation would promote macrophages to polarize into M2 type (6). In order to investigate how SOCS1 downregulation would affect the polarization of macrophages, the present study was conducted. First, shRNA targeting SOCS1 was screened. In the study, three SOCS1 shRNAs were designed, synthesized and cloned into pSilencer 2.1-U6 neo vector. Following transfecting shRNAs into mouse macrophage cells, SOCS1 expression was detected. Results indicated that only shRNA3 had a significant inhibitory effect, with the inhibition efficiency up to $85 \%$, while the inhibitory effect of the other two shRNAs was not obvious (Fig. 1B). SOCS1 expression was further detected by Western blot, and results showed that only shRNA3 could significantly inhibit SOCS1 expression, while the other two shRNAs did not substantially affect the protein levels of SOCS1 (Fig. 1C and D). Thus, shRNA3 has obvious effect of suppressing SOCS1 expression and it can be used for subsequent research.

Influence of SOCS1 downregulation and Xuebijing on the JAK/STAT pathway. The JAK/STAT signaling pathway is a stress response pathway that was only recently discovered $(14,15)$. It is widely involved in processes such as cell proliferation, differentiation, maturation, apoptosis and immune regulation, and is one of the many important ways for cytokine signal transduction $(14,15)$. As a main feedback regulatory protein of the JAK/STAT signaling pathway, SOCS1 conducts negative feedback inhibition $(5,16)$. After downregulating SOCS1 expression using shRNA, the mRNA levels of JAK1 and STAT1 both increased significantly. When STAT1 activation inhibitor, fludarabine, was added, the mRNA levels of SOCS1, JAK1 and STAT1 presented no significant changes (Fig. 2A). Meanwhile, western blotting indicated that, following the downregulation of SOCS1 expression, expression levels of JAK1, STAT1 and p-STAT1 increased significantly. Fludarabine can inhibit STAT1 phosphorylation and SOCS1 expression, but it cannot affect the expression of JAK1 and STAT1. In addition when SOCS1 shRNA transfection and fludarabine was added, the inhibition effect of fludarabine was weakened, but p-STAT1 expression remained upregulated (Fig. 2B and C). Furthermore, studies have reported that the compound traditional Chinese medicine, Xuebijing, can promote LPS to stimulate macrophages differentiation into M2 type (17). Therefore, the current study also explored the underlying mechanism of Xuebijing. Given that that mRNA and protein levels of SOCS1, JAK1 and STAT1 presented no significant changes when Xuebijing was administered (Fig. 2), Xuebijing may not serve its role through SOCS1, JAK1 and STAT1.

Influence of SOCS1 downregulation and Xuebijing on polarization of macrophages. Macrophages will polarize into M1 or M2 phenotypes under different conditions, and CCR7 (CD197) is the surface marker for M1 type cells, and CD206 is the surface marker for M2 type cells (18). Following the downregulation of SOCS1 expression, flow cytometry was conducted and the results are presented in Fig. 3. Results suggested that, following downregulation of SOCS1, the proportion of M1 type cells was significantly increased, while that of M2 type cells did not change significantly, indicating that downregulated SOCS1 expression is conducive to the polarization of macrophages into M1 phenotype. Adding 
A

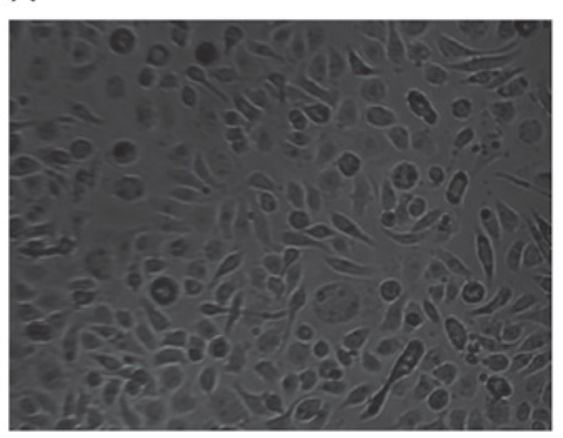

C

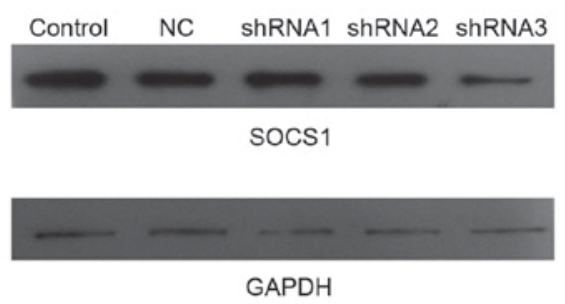

B

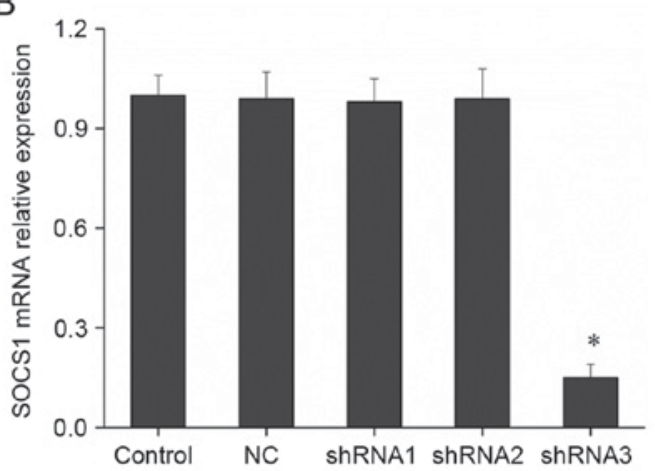

D

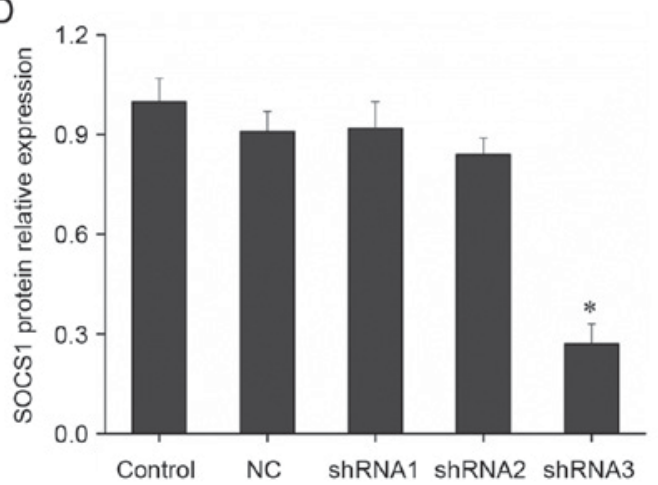

Figure 1. SOCS1 shRNA inhibitory effect. (A) Mouse macrophages were cultured in RPMI 1640 medium and images were captured at x200 magnification. (B) SOCS1 expression in macrophages following transfection with SOCS1 shRNA as measured by reverse transcription-quantitative polymerase chain reaction. (C and D) SOCS1 expression in macrophages following transfection with SOCS1 shRNA as measured by western blot analysis. Each bar represents the mean \pm standard deviation $(\mathrm{n}=3)$. ${ }^{*} \mathrm{P}<0.01$ vs. the control. SOCS1, suppressor of cytokine signaling-1; shRNA, short hairpin RNA; NC: negative control group that was transfected with the empty pSilencer 2.1-U6 neo vector. The control group was not transfected.

A

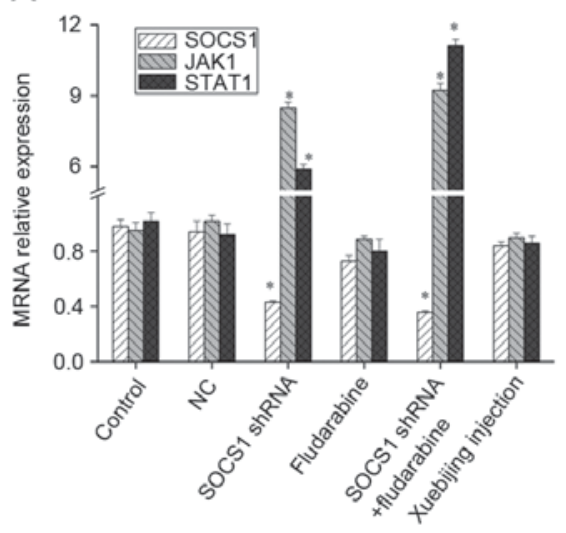

B

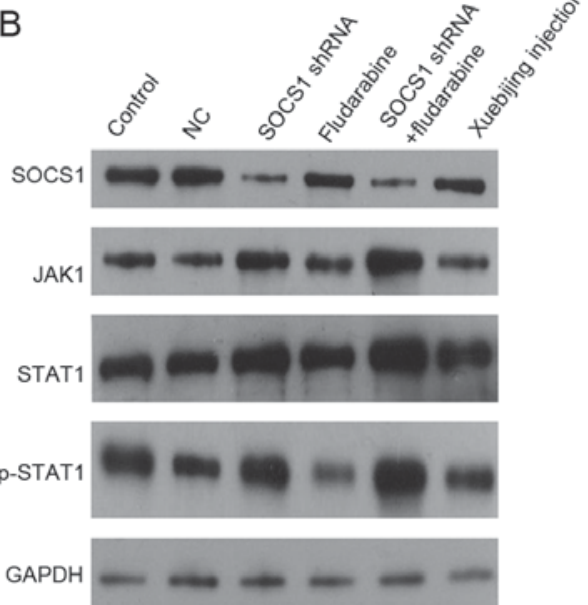

C

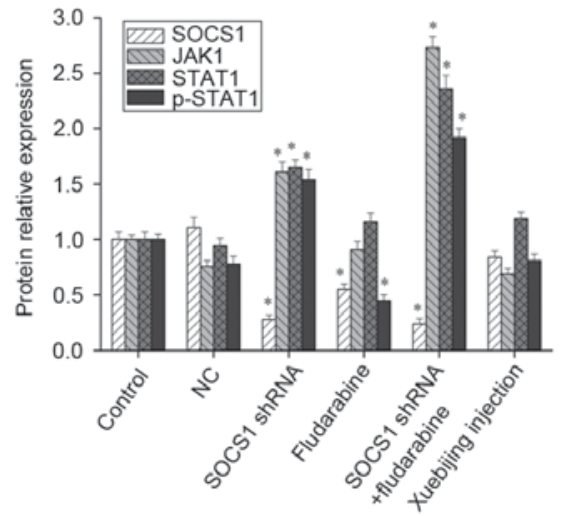

Figure 2. Expression of SOCS1, JAK1 and STAT1 in macrophages. Mouse macrophages were transfected with SOCS1 shRNA, added with fludarabine or Xuebijing, respectively, and then the expression of SOCS1, JAK1 and STAT1 was measured by (A) reverse transcription-quantitative polymerase chain reaction or (B and C) western blot analysis. Experiments were carried out at least in triplicate and the results were expressed as the mean \pm standard deviation. ${ }^{*} \mathrm{P}<0.01$ vs. control. SOCS1, suppressor of cytokine signaling-1; JAK1, Janus kinase 1; STAT1, signal transducer and activator of transcription; shRNA, short hairpin RNA; NC, negative control.

fludarabine or Xuebijing alone did not affect macrophage polarization. However, when SOCS1 shRNA transfection and fludarabine was administered, macrophages could polarize into M1 and M2 phenotypes, and the ratio of two types was significantly increased; but the proportion of M1 type cells slightly decreased while compared to the shRNA transfection group.
Influence of SOCS1 downregulation and Xuebijing on the expression of inflammatory cytokines. Changes in SOCS1 expression affect macrophage polarization, and different phenotypes of macrophages secrete and express different inflammatory cytokines. Activated M1 macrophages can induce expression of pro-inflammatory cytokines, such as TNF- $\alpha$ and IFN- $\gamma$, while activated M2 macrophages can induce high 

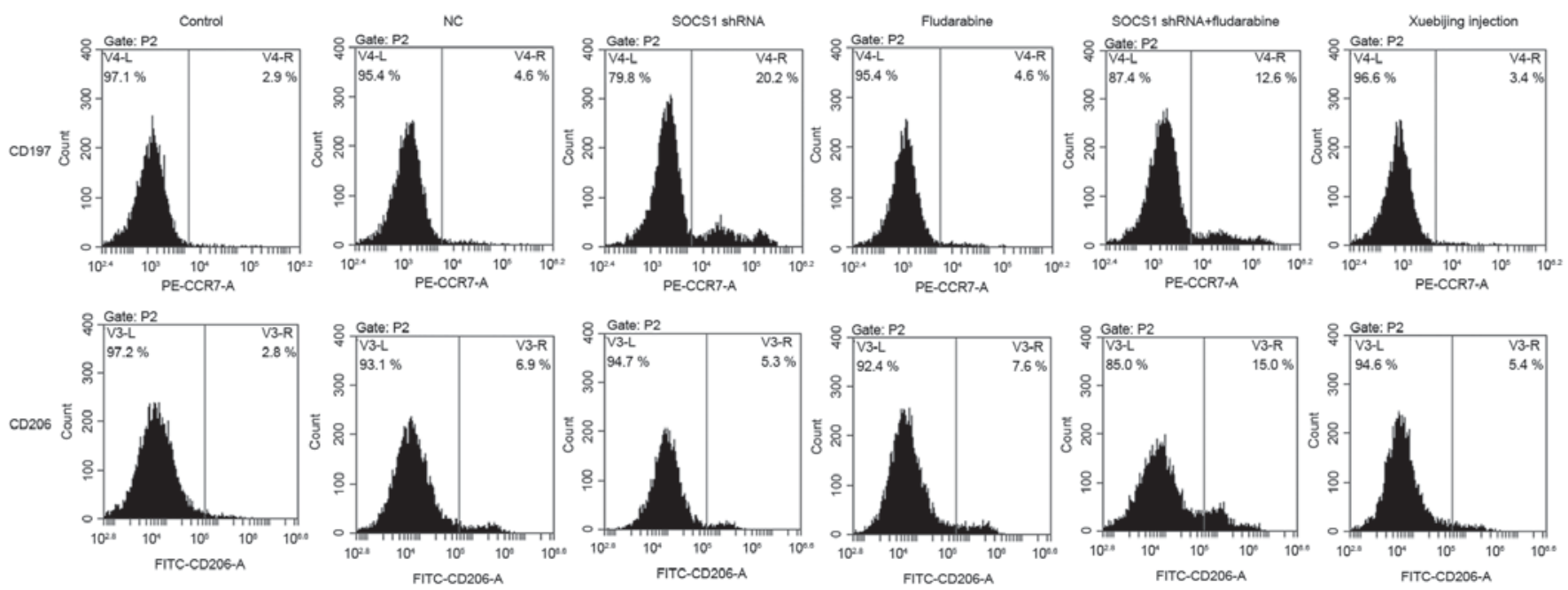

Figure 3. Macrophage activation and polarization. Mouse macrophages were transfected with SOCS1 shRNA, and either fludarabine or Xuebijing, was added. Macrophages were then stained with fluorescently-labeled monoclonal antibodies specific for CD206 and CCR7 and analyzed by flow cytometry. NC, negative control; SOCS1, suppressor of cytokine signaling-1; PE, phycoerythrin.
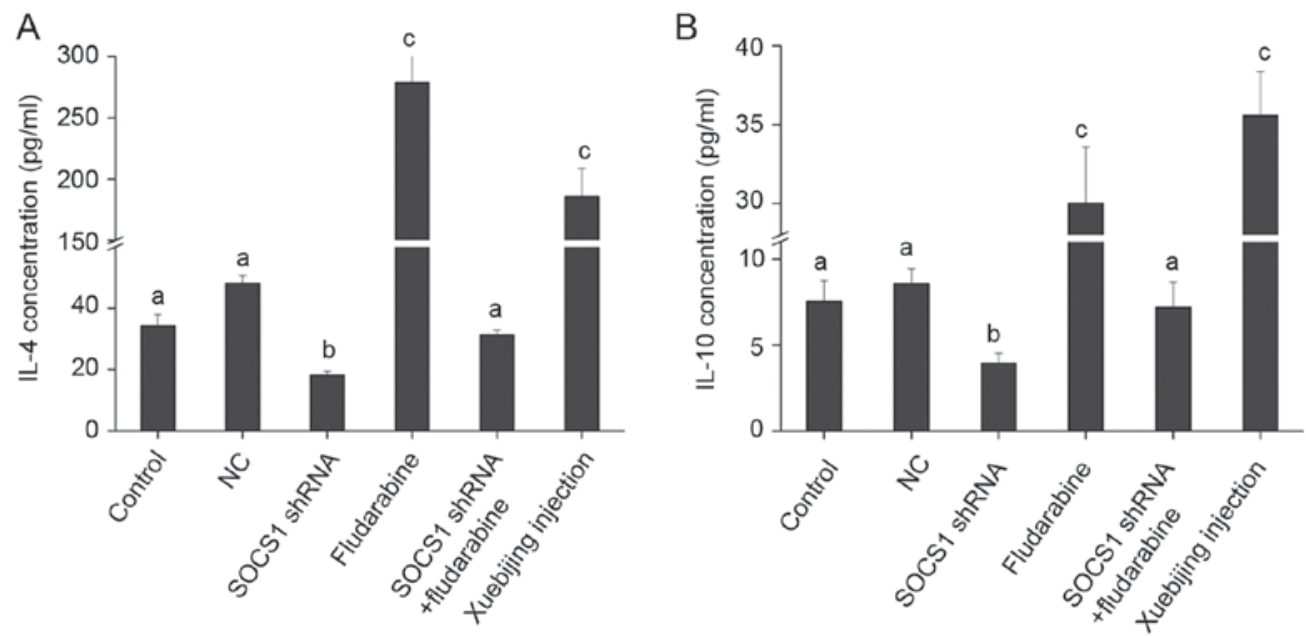

C

$\mathrm{D}$
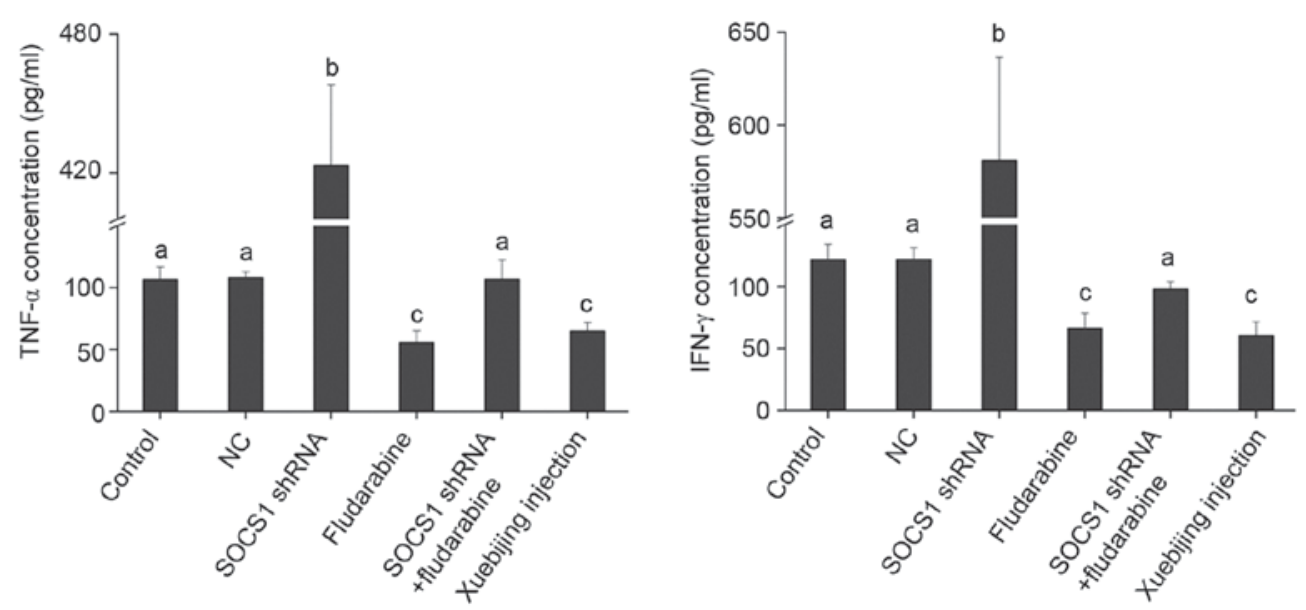

Figure 4. Supernatant (A) IL-4, (B) IL-10, (C) TNF- $\alpha$ and (D) IFN- $\gamma$ levels were detected by ELISA. Bars with different characters are statistically different to each other $(\mathrm{P}<0.01)$. IL, interleukin; TNF, tumor necrosis factor; IFN, interferon; NC, negative control; SOCS1, suppressor of cytokine signaling-1.

expression of anti-inflammatory cytokines, such as IL-4 and IL-10 (19). The present study attempted to detect the influence of SOCS1 on inflammatory cytokine expression. ELISA results indicated that, when downregulating SOCS1 expression, IL-4 and IL-10 expression was downregulated and TNF- $\alpha$ and IFN- $\gamma$ expression was significantly upregulated (Fig. 4), indicating that 
inhibited SOCS1 expression is not conducive to the secretion of anti-inflammatory cytokines, but is conducive to the secretion of pro-inflammatory cytokines. These findings are consistent with the result that, when SOCS1 expression is inhibited, macrophages polarize into M1 type (Fig. 3). Furthermore, when adding fludarabine or Xuebijing, IL-4 and IL-10 expression significantly increased, and TNF- $\alpha$ and IFN- $\gamma$ expression significantly decreased. When SOCS1 shRNA transfection and fludarabine was added, expression of IL-4, IL-10, TNF- $\alpha$ and IFN- $\gamma$ reported no significant changes (Fig. 4), indicating that the two have a mutually antagonistic effect.

\section{Discussion}

Macrophage polarization is involved in various signaling pathways and in the action of transcription factors, and polarized macrophages are associated with the occurrence and development of many diseases $(1,2)$. Therefore, research on the plasticity mechanism of macrophages polarization is of great significance. In addition, research on macrophage polarization can give us a better understanding of the mechanisms of cells, especially immune cells adapting to different micro-environments, and serve as important theoretical basis for treatment various diseases.

SOCS is generated under inducement of cytokines. As a crucial negative regulatory protein of the JAK/STAT signaling pathway, it regulates the body's over-stimulation for cytokines by inhibiting signal transduction of cytokines (5). Previous studies have indicated that SOCS is an important physiological regulator of the innate and adaptive immune response; it can promote or inhibit activation of macrophages and dendritic cells, participate in $\mathrm{T}$ cells differentiation and immune regulation $(5,16)$. SOCS1 is an important member, and there have been some progress in the study of its function at present, but there were few studies about the effect of its downregulation on macrophages. In the present study, shRNA targeting SOCS1 was screened. Results demonstrated that, among the three synthesized shRNAs, one had a significant inhibitory effect, with the inhibition efficiency up to $85 \%$, and western blot detection also demonstrated that it could significantly inhibit the expression of SOCS1. Therefore, this shRNA can be used for subsequent research.

SOCS regulates the intensity and duration of cytokines, hormones and growth factors primarily through inhibition of JAK/STAT signal transduction (5). Through the JAK/STAT pathway, macrophages will make appropriate response to $>20$ types of cytokine (20). Therefore, the influence of SOCS1 on the JAK/STAT pathway was first detected. Following downregulating SOCS1 expression in macrophages using shRNA, expression levels of JAK1 and STAT1 both increased significantly, and phosphorylation levels of STAT1 also increased significantly. Fludarabine did not affect the mRNA levels of SOCS1, JAK1 and STAT1, but it could downregulate protein levels of SOCS1 and inhibit phosphorylation of STAT1. Furthermore, when Xuebijing was administered, the mRNA and protein levels of SOCS1, JAK1 and STAT1 had no significant changes, indicating that it may not play its role through SOCS1, JAK1 and STAT1. SOCS1 was previously indicated to act on the JH1 domain of JAKs, inhibiting the kinase activity, and thereby inhibited the conduction of JAK/STAT signaling pathway (21). The current study demonstrated that SOCS1 downregulation is conducive to the activation of the JAK/STAT pathway. But SOCS is not only a negative regulator for JAK/STAT signaling pathway but also a target gene of the pathway. Therefore, the result that fludarabine could downregulate SOCS1 expression is consistent with current findings.

Further flow cytometry detection demonstrated that, after the downregulation of SOCS1 expression, the proportion of M1-type cells significantly increased, while that of M2-type cells did not change significantly. Adding fludarabine or Xuebijing alone did not affect macrophage polarization. However, when SOCS1 shRNA transfection and fludarabine was added, macrophages could polarize into M1 and M2 phenotypes, but the proportion of M1 type cells slightly decreased while compared to the shRNA transfection group. It indicated that downregulated SOCS1 expression is conducive to the polarization of macrophages into the M1 phenotype, while fludarabine had an inhibitory effect. Studies on mice knocked out of SOCS1 also suggested that SOCS1 has an inhibitory effect on STAT1 (22). Activated M2 macrophages in vitro demonstrated a selective and IL-4-dependent upregulation of SOCS1 (6). The test using small interfering RNA to knockout SOCS1 in bone marrow-derived macrophage cells indicated that SOCS1 expression is of great importance for IL-4-induced M2-type characteristics (6). Spence et al (23) stimulated the mice LPS to knockdown SOCS2 and SOCS3, and reported that SOCS2 and SOCS3 are important regulatory molecules for polarization into M1 and M2 type macrophages and inflammatory responses. The SOCS2 knockout macrophages highly expressed M1-type markers, while SOCS3 knockout macrophages highly expressed M2-type markers, indicating that SOCS2 plays an important role in polarization into M2 type macrophages and inhibition on inflammatory responses. However, while SOCS3 is involved in polarization into M1 type macrophages and promotes the occurrence of inflammatory responses (8). The present study and above results all demonstrate that SOCS1 serve a key role in the regulation of macrophage polarization.

Different phenotypes of macrophages secrete and express various inflammatory cytokines, so ELISA was used to further detect the expression of iconic factors such as IL-4, IL-10, TNF- $\alpha$ and IFN- $\gamma$. Results demonstrated that, after SOCS1 expression was downregulated, expression of IL-4 and IL-10 was downregulated, while expression of TNF- $\alpha$ and IFN- $\gamma$ was significantly upregulated. When adding fludarabine or Xuebijing, expression of IL-4 and IL-10 significantly increased, while that of TNF- $\alpha$ and IFN- $\gamma$ significantly decreased. When SOCS1 shRNA was transfected and fludarabine was added, expression of above factors reported no significant changes. The above results indicated that inhibited SOCS1 expression is not conducive to the secretion of anti-inflammatory cytokines, but is conducive to the secretion of pro-inflammatory cytokines, and fludarabine had the role of blocking the function of SOCS1 shRNA. Studies have suggested that SOCS1 serves an important role in the negative feedback regulation of the JAK2/STAT3 signaling pathway $(5,24)$. It was further indicated that SOCS1 inhibited JAK2 phosphorylation through the central SH-2 region and inhibited activation of the STAT3 signaling pathway, thus regulating the secretion of inflammatory cytokines by macrophages $(25,26)$. Function loss of 
SOCS1 may lead to JAK2/STAT3 activation and cytokine accumulation. In contrast, an increase in SOCS1 expression would inhibit JAK2/STAT3 activation and reduce secretion of cytokines $(27,28)$. Therefore, downregulation of SOCS1 could activate the JAK/STAT pathway and thereby promote polarization of macrophages into M1 type.

In summary, changes in SOCS1 expression serve an important regulatory role in macrophage polarization. With a clear understanding of its polarization mechanism, we will be able to intervene reasonably certain key steps of macrophage polarization to reverse the imbalance of macrophage polarization, and thereby treat various immune-related diseases from new angles.

\section{Acknowledgements}

The present study was supported by grants from Science and Technology Planning Project of Guangdong Province (grant no. 2012B031800291), the Science and Technology Planning Project of Guangzhou City (grant no. 201300000160). This study was supported by funding from Tianjin Chase Sun Pharmaceutical Co., Ltd.

\section{References}

1. Zhou D, Huang C, Lin Z, Zhan S, Kong L, Fang C and Li J: Macrophage polarization and function with emphasis on the evolving roles of coordinated regulation of cellular signaling pathways. Cell Signal 26: 192-197, 2014.

2. Murray PJ, Allen JE, Biswas SK, Fisher EA, Gilroy DW, Goerdt S, Gordon S, Hamilton JA, Ivashkiv LB, Lawrence T, et al: Macrophage activation and polarization: Nomenclature and experimental guidelines. Immunity 41: 14-20, 2014.

3. Verreck FA, de Boer T, Langenberg DM, Hoeve MA, Kramer M, Vaisberg E, Kastelein R, Kolk A, de Waal-Malefyt R and Ottenhoff TH: Human IL-23-producing type 1 macrophages promote but IL-10-producing type 2 macrophages subvert immunity to (myco)bacteria. Proc Natl Acad Sci USA 101: 4560-4565, 2004.

4. Bouhlel MA, Derudas B, Rigamonti E, Dièvart R, Brozek J, Haulon S, Zawadzki C, Jude B, Torpier G, Marx N, et al: PPARgamma activation primes human monocytes into alternative M2 macrophages with anti-inflammatory properties. Cell Metab 6: 137-143, 2007.

5. Tamiya T, Kashiwagi I, Takahashi R, Yasukawa H and Yoshimura A: Suppressors of cytokine signaling (SOCS) proteins and JAK/STAT pathways: Regulation of T-cell inflammation by SOCS1 and SOCS3. Arterioscler Thromb Vasc Biol 31: 980-985, 2011.

6. Whyte CS, Bishop ET, Rückerl D, Gaspar-Pereira S, Barker RN, Allen JE, Rees AJ and Wilson HM: Suppressor of cytokine signaling (SOCS)1 is a key determinant of differential macrophage activation and function. J Leukoc Biol 90: 845-854, 2011.

7. Stoiber D, Kovarik P, Cohney S, Johnston JA, Steinlein P and Decker T: Lipopolysaccharide induces in macrophages the synthesis of the suppressor of cytokine signaling 3 and suppresses signal transduction in response to the activating factor IFN-gamma. J Immunol 163: 2640-2647, 1999.

8. Liu Y, Stewart KN, Bishop E, Marek CJ, Kluth DC, Rees AJ and Wilson HM: Unique expression of suppressor of cytokine signaling 3 is essential for classical macrophage activation in rodents in vitro and in vivo. J Immunol 180: 6270-6278, 2008.

9. Arnold CE, Whyte CS, Gordon P, Barker RN, Rees AJ and Wilson HM: A critical role for suppressor of cytokine signalling 3 in promoting M1 macrophage activation and function in vitro and in vivo. Immunology 141: 96-110, 2014.
10. Prêle CM, Woodward EA, Bisley J, Keith-Magee A, Nicholson SE and Hart PH: SOCS1 regulates the IFN but not NFkappaB pathway in TLR-stimulated human monocytes and macrophages. J Immunol 181: 8018-8026, 2008.

11. He XD, Wang Y, Wu Q, Wang HX, Chen ZD, Zheng RS, Wang ZS, Wang JB and Yang Y: Xuebijing protects rats from sepsis challenged with Acinetobacter baumannii by promoting Annexin A1 expression and inhibiting proinflammatory cytokines secretion. Evid Based Complement Alternat Med 2013: 804940, 2013.

12. Liu X, Hu Z, Zhou B, Li X and Tao R: Chinese herbal preparation Xuebijing potently inhibits inflammasome activation in hepatocytes and ameliorates mouse liver ischemia-reperfusion injury. PLoS One 10: e0131436, 2015.

13. Livak KJ and Schmittgen TD: Analysis of relative gene expression data using real-time quantitative PCR and the 2(-Delta Delta C(T)) method. Methods 25: 402-408, 2001.

14. Sprague AH and Khalil RA: Inflammatory cytokines in vascular dysfunction and vascular disease. Biochem Pharmacol 8: 539-552, 2009.

15. Murray PJ: The JAK-STAT signaling pathway: Input and output integration. J Immunol 178: 2623-2629, 2007.

16. Yoshimura A: Regulation of cytokine signaling by the SOCS and Spred family proteins. Keio J Med 58: 73-83, 2009.

17. Liu YC, Yao FH, Chai YF, Dong N, Sheng ZY and Yao YM: Xuebijing injection promotes M2 polarization of macrophages and improves survival rate in septic mice. Evid Based Complement Alternat Med 2015: 352642, 2015.

18. Chen Z, Li F, Yang W, Liang Y, Tang H, Li Z, Wu J, Liang H and Ma Z: Effect of rTsP53 on the M1/M2 activation of bone-marrow derived macrophage in vitro. Int J Clin Exp Pathol 8: 13661-13676, 2015.

19. Ouedraogo R, Daumas A, Ghigo E, Capo C, Mege JL and Textoris J: Whole-cell MALDI-TOF MS: A new tool to assess the multifaceted activation of macrophages. J Proteomics 75: 5523-5532, 2012.

20. Hu X, Chen J, Wang L and Ivashkiv LB: Crosstalk among Jak-STAT, toll-like receptor and ITAM-dependent pathways in macrophage activation. J Leukoc Biol 82: 237-243, 2007.

21. Krebs DL and Hilton DJ: SOCS proteins: Negative regulators of cytokine signaling. Stem Cells 19: 378-387, 2001.

22. Wormald S and Hilton DJ: Inhibitors of cytokine signal transduction. J Biol Chem 279: 821-824, 2004.

23. Spence S, Fitzsimons A, Boyd CR, Kessler J, Fitzgerald D, Elliott J, Gabhann JN, Smith S, Sica A, Hams E, et al: Suppressors of cytokine signaling 2 and 3 diametrically control macrophage polarization. Immunity 38: 66-78, 2013.

24. Davey GM, Heath WR and Starr R: SOCS1: A potent and multifaceted regulator of cytokines and cell-mediated inflammation. Tissue Antigens 67: 1-9, 2006.

25. Tajiri K, Imanaka-Yoshida K, Matsubara A, Tsujimura Y, Hiroe M, Naka T, Shimojo N, Sakai S, Aonuma K and Yasutomi Y: Suppressor of cytokine signaling 1 DNA administration inhibits inflammatory and pathogenic responses in autoimmune myocarditis. J Immunol 189: 2043-2053, 2012.

26. Hashimoto M, Ayada T, Kinjyo I, Hiwatashi K, Yoshida H, Okada Y, Kobayashi T and Yoshimura A: Silencing of SOCS1 in macrophages suppresses tumor development by enhancing antitumor inflammation. Cancer Sci 100: 730-736, 2009.

27. Capello D, Gloghini A, Baldanzi G, Martini M, Deambrogi C, Lucioni M, Piranda D, Famà R, Graziani A, Spina M, et al: Alterations of negative regulators of cytokine signalling in immunodeficiency-related non-Hodgkin lymphoma. Hematol Oncol 31: 22-28, 2013

28. Souma Y, Nishida T, Serada S, Iwahori K, Takahashi T, Fujimoto M, Ripley B, Nakajima K, Miyazaki Y, Mori M, et al: Antiproliferative effect of SOCS-1 through the suppression of STAT3 and p38 MAPK activation in gastric cancer cells. Int J Cancer 131: 1287-1296, 2012 